\title{
Measurement approaches to the sense of humor: Introduction and overview
}

\author{
Ruch, Willibald
}

\begin{abstract}
There has been a renaissance of research interest in the "sense of humor" in recent years, partly äs an attempt to define the concept but more strenuously to provide Instruments for its measurement. A quick count of recent publications shows an average of two to three new sense of humor- instruments per year - or one every four to six months. This intensity of research is unparalleled in the history of humor research and contrasts sharply with 25 years ago when the renewal of interest in humor feil into a period where cognitive approaches dominated the Zeitgeist in psychology, and the long tradition of personality research in humor was at a point of Stagnation. As an indicator, the "classic" anthologies Psychology of Humor (1972, edited by Jeffrey Goldstein and Paul McGhee) and Humor and Laughter (1976, edited by Tony Chapman and Hugh Foot) contained no chapter on personality and humor. Perhaps even more striking, "sense of humor" did not appear among the index terms in the former. In the latter, the index guides the reader to Lawrence LaFave's suspicion that the sense of humor is merely a "myopic illusion" (LaFave, Haddad, and Maesen 1976: 79).
\end{abstract}

DOI: https://doi.org/10.1515/humr.1996.9.3-4.239

Posted at the Zurich Open Repository and Archive, University of Zurich ZORA URL: https://doi.org/10.5167/uzh-77692

Journal Article

Published Version

Originally published at:

Ruch, Willibald (1996). Measurement approaches to the sense of humor: Introduction and overview. HUMOR: International Journal of Humor Research, 9(3-4):239-250.

DOI: https://doi.org/10.1515/humr.1996.9.3-4.239 


\section{Measurement approaches to the sense of humor: Introduction and overview*}

WILLIBALD RUCH

There has been a renaissance of research interest in the "sense of humor" in recent years, partly as an attempt to define the concept but more strenuously to provide instruments for its measurement. A quick count of recent publications shows an average of two to three new sense of humorinstruments per year - or one every four to six months. This intensity of research is unparalleled in the history of humor research and contrasts sharply with 25 years ago when the renewal of interest in humor fell into a period where cognitive approaches dominated the zeitgeist in psychology, and the long tradition of personality research in humor was at a point of stagnation. As an indicator, the "classic" anthologies Psychology of Humor (1972, edited by Jeffrey Goldstein and Paul McGhee) and Humor and Laughter (1976, edited by Tony Chapman and Hugh Foot) contained no chapter on personality and humor. Perhaps even more striking, "sense of humor" did not appear among the index terms in the former. In the latter, the index guides the reader to Lawrence LaFave's suspicion that the sense of humor is merely a "myopic illusion" (LaFave, Haddad, and Maesen 1976: 79).

The renaissance of research on the sense of humor: Where to?

Apparently, humor researchers were not discouraged by that and picked up the issue again. However, this renewed research interest raises several questions such as what do we need a sense of humor construct for, what is the research agenda, and what decisions regarding structural characteristics need to be made? I will briefly discuss these questions and then address the problems associated with the trait-label. 
Do we need humor as a personality variable and if so, what for?

Everyday experience as well as systematic assessment tells us that individuals vary with respect to humor phenomena. Some of that variation is temporal, for example, due to different moods or mental states; however, an individual's humor style is also stable that is, certain behaviors occur habitually in certain individuals and not in others. In language, humor type nouns, like wit, wag, humorist, cynic, or jester, and humor-related trait-adjectives, like humorous, whimsical, witty, jocular, or cynical demonstrate that habitual individual differences in humor are so apparent and so significant that words were invented for them. These facts provide ample justification for a personality-approach to humor which should describe, predict, and eventually explain the humor-related differences among people. ${ }^{1}$

What is the research agenda?

The research agenda, therefore, is to describe the entirety of observable habitual individual differences in humor, investigate their interrelations in a systematic way, and eventually define a smaller set of traits that account for the differences observed, that is, that make up a person's "sense of humor." Logically, the steps are to explore the nature of these dimensions; describe the full range of variations within these dimensions, and develop measurement instruments. Then the relationships among these dimensions of humor as well as their relation to other dimensions of personality need to be studied.

Theoretical models should be developed which allow the prediction of individual differences in humor-relevant situations as well as other domains of behavior. Results from such studies should be used to refine the models. The causes of individual differences in humor need to be studied considering both environmental and heredity factors.

Group differences are of interest, too, be they gender, class, regional, or national differences. We also need information on intraindividual differences (or intrapsychic changes), whether short-lived or related to the entire life-span as well as how an individual's sense of humor can be changed permanently and by what sorts of factors. Obviously, there is still a long way to go before these and related questions are answered. 
How do sense of humor-concepts differ? Variations in formal features

There are many fundamentally different approaches to personality which could serve as a formal basis for the study of humor. However, as in general empirical personality research, currently most humor researchers utilize the trait approach. Even in this sort of approach, considerable variations exist, and the type of sense of humor-concept put forward will be very different depending on the position one takes in a variety of fundamental issues.

One issue pertaining to how models may differ relates to the concept's generality: Is the humorous personality described at the level of habits or specific behavior tendencies or at a higher level of abstraction? Put another way, are we interested in distinguishing among many but narrow components, or do we prefer a few broad traits (or even a very global unidimensional concept)? Another issue relates to the structure of the components of the sense of humor: Should these traits be orthogonal, or is it more reasonable to assume the components of humor to be correlated? Is the sense of humor, or components thereof, unipolar (for example, not humorous versus very humorous) or bipolar (for example, serious versus humorous)? In other words, are individuals on the low end of a continuum lacking the trait typical for the high-scorers, or are they characterized by high scores on an opposite trait? Are all components of the sense of humor referring to typical behavior, or temperament, or is there such a thing as maximal behavior, or ability? Do we treat any sort of humor behavior as equal (that is, there is no right or wrong), or do we evaluate them according to some criteria? (The answers to the last two questions, of course, determine the nature of the assessment tool required; is a self-report scale valid, or does it need to be a behavioral test?)

More importantly, should the approach be a comprehensive account of the sense of humor, or should it represent just one or more isolated components, and if it is intended to be comprehensive, what realm should it cover? Does it include negative aspects like the disposition for laughing at, ridiculing, sarcasm, or other socially undesirable forms of humor? Comprehensiveness at the trait-level requires comprehensiveness, or representativeness at the level of trait-indicators, i.e., the observable humor behavior that finally is studied to derive the concepts. How can we achieve this? What strategy can guarantee that we will adequately define the realm of phenomena to be studied? Finally, what structural model should be applied to represent the trait-indicators? For example, can they be expected 
to be located on dimensions (perhaps of a hierarchical nature), or would a circular arrangement fit their intrinsic structure better? Thus, humor researchers will have to decide on a variety of issues about which formal features best suit the current realm.

\section{Is "sense of humor" a good representative of humor at the trait level?}

So far I have treated the expression "sense of humor" as the only or best label to describe habitual individual differences in humor-related behavior. Of course, "sense of humor" is the expression one comes up with immediately; it is used both in everyday conversation and in technical reports, and some scales carry this label as well. However, one has to add that there is also no agreement either in research or in everyday language about what this expression exactly is supposed to mean. The reasons for this are manifold and stem from the confusion in both proposed terminological and laypersons' use of the core term humor. There are other problems associated with the use of the term "sense of humor" which I will address as well.

How narrow or broad is the scope of "humor"?

One sort of confusion is that humor is used both as a super- and subordinate concept. For example, one tradition sees humor at the same level as wit, satire, irony, or nonsense, as part of the comic, which is one category of aesthetics (and discriminated from others, like tragedy, beauty, or harmony). Here, humor has a very narrow and somewhat precise definition (as a world view allowing one to perceive and enjoy incongruities stemming from imperfections of life and fellow human in a benevolent way) shaped over the centuries. In this context, it is not only legitimate but also necessary to distinguish among the dispositions. Thus, the "sense of humor" needs to be distinguished from the "sense of fun," "sense of wit," "sense of ridicule," "sense of comic," and others.

However, there are also the traditions that place humor and the comic (and jokes) at the same level (for example Freud 1905) or that place humor at a superordinate level; to see it as a catch-all for all phenomena of this field. It is the view adopted by current Anglo-American humor research (even without an extensive definitional process) that there is a de facto agreement to use humor as the umbrella-term for all phenomena related 
to the comic, wit, laughter, and humor (in its narrow sense). This is most significantly expressed in the name of the present journal. From this perspective, "sense of humor" is indeed a good candidate for labeling humor as a personality trait.

\section{Sense of humor as a disposition for teasing and laughing at others?}

However, if we want to use "sense of humor" as an umbrella-term for humor as a personality trait, we have to explicitly exempt this expression from its benevolent, philanthropic connotations. Given that the trait we want to develop should be descriptive of all forms of humor behavior (not only the prosocial ones), the sense of humor should also represent the disposition for less valued humor phenomena, such as sarcasm, mocking, ridicule, satire, or irony. In fact, this proposed use of "sense of humor" conflicts with its often-quoted "social desirability."

We have to ask, however, whether the positive value is "inherent" in the sense of humor or whether it acquired this value, and if this is the case, when and how? Schmidt-Hidding (1963) meticulously pointed out for several languages (German, English, and Spanish) the many transitions in the meaning of the term humor from the Latin word for liquid, fluid, to its current usage. From this it is apparent that (a) humor (like wit, which before meant reason, mind, accumulated knowledge) did not enter the field of the comic before the late 16th century; (b) while wit soon acquired its definite meaning, this process was much longer and less straightforward for humor; (c) the term humor (unlike other terms used by the common people, like jest or wag) was primarily used by the educated, and writers and critics were involved in clarifying its meaning; and (d) these attempts were not always guided only by the wish for precision of language but also loaded by world views and values (in particular by humanistic ideas).

Indeed, humanism perhaps brought the significant shift in the meaning of humor. By the end of the 17th century, people became weary of "putdown" witticisms. People should not be laughed at because of peculiarities of temperament, it was argued, since they were not responsible for them. A term became necessary for the humanitarian, tolerant, and benevolent forms of laughter, and that term was found in good humor, later humor (Schmidt-Hidding 1963). Still, it was considered permissible to laugh at the pompous, the unreal, the faked, the conceited, etc. Of course even a 
serious person can hold attitudes, views, statements, etc. which are ridiculous. A "test of ridicule" would put these to the test. Good humor denoted the sovereign attitude exposing oneself to such a test, and passing it, like having wit, was considered to be a talent. Schmidt-Hidding notes this may have been the origin of the notion of the "sense of humor" although this expression was not yet in use.

Thus, it is not surprising (but simply tautological) that we now perceive the sense of humor to be valuable and socially desirable. In terms of research, though, the term is value-laden; it is not a descriptive but a prescriptive term. As an enduring consequence of this intended restriction, we now hesitate to subsume negative features under the scope of this concept. Is it possible to use "sense of humor" as a descriptive technical term, without transporting those unwanted historical connotations? This is an issue $I$ believe we need to consider.

The utility and limitations of "sense of humor" for uni- and multidimensional models

Depending on the dimensionality of habitual individual differences in humor, "sense of humor" will either refer to a content-saturated concept (a g-factor of humor) or merely be a collective noun for the entirety of these dimensions and, when applied to individuals, refer to the personal profile in these dimensions. These differences may best be exemplified by the characteristic structure of the respective research programs, which are one- or two-step.

In case of unidimensionality, the research program demands designing an assessment tool and developing and empirically testing a theoretical model of the sense of humor which eventually can be refined on the basis of such studies. Here "sense of humor" refers to a homogeneous trait with some descriptive or even explanatory power. In the more likely case of a multidimensionality of the sense of humor (more precisely, when there is no g-factor at a hierarchically higher level), a further type of inquiry needs to be added: What are the components of the sense of humor, and how are they interrelated?

Sense of humor $=f\left(X_{1}, X_{2}, X_{3}, \ldots X_{i}, \ldots X_{n}\right)$

Thus, we are asking how many $(n)$ dimensions $\left(X_{i}\right)$ do we need to fully account for all habitual differences in humor, and what might the internal 
structure of these dimensions be? Here, "sense of humor" just serves as a category label for a class of traits (like temperament, intelligence, and emotion) and by itself has no predictive or explanatory power. While the other type of question remains, it is applied to the individual components of the sense of humor (which carry the theoretical substance), not the sense of humor itself. Thus, we develop and test models for each of the components, and the results tell us something about the particular component of sense of humor (but not about the sense of humor per se).

The two uses of sense of humor as a trait category label and a homogeneous construct should not be confused because (a) hypotheses cannot be derived and tested for the global sense of humor category (only for its components); (b) individual scales cannot be used to represent the "sense of humor" (at least not until the first type of question delineated above has been answered), but only particular components; and (c) research findings are restricted in validity to the particular component assessed and should not be expanded to other components or to the sense of humor per se. Confusing these levels can have serious consequences. Studies are prone to fail if we test even valid hypotheses with the wrong instruments; for example, one component of humor might predict facial expressiveness in response to humorous stimuli (but not the cognitive processes involved), and the other might account for the cognitive processes but not for the intensity of the affective response.

Thus, while "sense of humor" might be an appropriate term in both cases, its meaning varies with the context of dimensionality, and we need to know and consider the limitations in its potential usage.

Quotidian versus technical uses of the term "sense of humor":

What is their relationship?

Humor research also needs to study the layperson's concept and use of the term in everyday conversation. It probably does not cover the whole domain of humor and means different things for different people. (One of the articles in this issue gives answers to this question, alerting us to the necessity to explicitly distinguish between the technical and everyday usages of the term.) There is some conflict, though, between the use of the expression "sense of humor" in items of questionnaires and their aim to measure the "sense of humor" as a technical concept. Obviously, research participants will understand the term in the lay sense (with, no doubt, 
considerable variations); but their answers end up as scores on the technical side, which could be problematic if the two meanings are grossly different. Thus, while self- and peer-ratings of "sense of humor" may be used as criteria in validating the scales, the problem remains as to whether selfattributions of "having a sense of humor" should serve as trait-indicators, and be used as items in a questionnaire.

In general, it seems that we will have to be more explicit regarding the construction of the concept, its formal features, and the selection and use of the trait-label, be it "sense of humor" or not.

\section{The papers}

Some of the papers in this special issue were taken from the symposium Approaches to the Sense of Humor: Concepts and Measurement I organized for the 1994 International Society for Humor Studies Conference in Ithaca, New York, which was intended to focus attention on this renewed research interest in the sense of humor as well as to provide a forum for the presentation and discussion of new and revised individual inventories, reviews of accumulated research around existing instruments, methodological studies, and the study of the sense of humor in relation to various phenomena.

Symposium presentations as well as other submissions were initially considered for inclusion in this special issue. It was not easy to find a compromise between representing the current research activity and the space restrictions inevitably given by the size of a single issue of the journal. So, unavoidably, the picture must be incomplete. What is missing? For example, there is an overrepresentation of self-report scales at the expense of behavioral tests and peer-evaluations mainly because there are no newly constructed inventories of that kind. However, the study by Köhler and Ruch covers behavioral measures as well as a variety of self-report scales not separately presented here for one reason or another. For example, there will be a new inventory by Beth Manke, Judy Dunn, and Robert Plomin (personal communication), but a manuscript describing its construction was not completed at the time the issue went to press. Nevertheless, the articles included represent different approaches to the field including those of high current interest.

The article by Rod Martin gives an overview of research conducted with two widely used humor questionnaires developed by him and Herbert 
Lefcourt. Although not the first inventories in the field of self-reports, Martin and Lefcourt's Situational Humor Response Questionnaire (SHRQ) and the Coping Humor Scale (CHS) were most influential. For once, this line of inquiry most consequently pushed forward the importance of studying the sense of humor as a genuine personality trait. Furthermore, a new direction in empirical humor research was established which was aimed at studying the moderating role of the sense of humor in the impact of life stress on mood disturbances.

The review informs about the development of the scales, including information about their reliability in the original and replication studies, and provides an up-to-date account of the validity of the scales based on empirical studies conducted in a number of different countries. These findings relate to (a) validity in the field of humor (i.e., how it correlates with humor-related behavior as assessed by other tests, experimental variables, and self- and peer-evaluations); (b) the 'humor as a stress-moderator' hypothesis; (c) the relationship with coping-related variables; and (d) the factor space occupied by the measures. The review involves a refinement of the hypotheses and arrives at a general picture of the constructs measured.

The article by Kenneth H. Craik, Martin D. Lampert, and Arvalea Nelson presents their approach to the assessment of humor (i.e., the Humorous Behavior Q-sort Deck-HBQD) and applies it to a variety of fundamental research questions. The measurement approach underlying the HBQD is characterized by three elements: (a) the focus on the assessment of humorrelated behaviors or behavior tendencies and, when aggregated, styles of humorous conduct; (b) the attempt to cover the whole behavioral domain of everyday humorous conduct as comprehensively as possible rather than formulating partly redundant items for the assessment of a few selected traits or components of humor; and (c) the application of the Q-sort technique to the assessment of humor rather than using conventional scales. The Q-sort provides an ipsativized profile of humor, i.e., a standardization within each individual.

The research presented systematically examines the relations between the vague folk concept of "sense of humor" (globally assessed) and the concept of "styles of humor" by relating them to each other (at the level of HBQD-items and HBQD-factors) but also to social desirability, the Jungian concept of extraversion-introversion, and a broad range of personality variables as assessed by an omnibus personality inventory. The results clearly demonstrate the merits of a comprehensive approach to the 
assessment of individuals' understanding of humor and their styles of everyday humorous conduct and underscores the limitations of the use of a global rating of the vague and undifferentiated notion of "sense of humor."

The article by Willibald Ruch, Gabriele Köhler, and Christoph van Thriel looks at humor from a temperament perspective and summarizes the state of the art in the construction of the inventory of the traits and states delineated in their model. The three characteristic features in this approach are (a) the assumptions that there are temperamental traits underlying individual differences in humor-related phenomena; (b) both trait and state dispositions of humor behavior and experience are considered; and (c) in addition to dispositions for acting and reacting with humor, factors presumably accounting for forms of humorlessness are considered as well. While the concepts presented have a long history in temperament research, both their current definition (with the help of facets) and their utility in humor research have to be substantiated in validity studies. That article focuses on the description and application of the construction strategy and presents the psychometric characteristics of two resulting forms of the trait version of the inventory. Regarding validity, only matters of construct validity are touched upon; first, the homogeneity of the facets is examined, and second, the convergence of self- and peer-evaluation is studied.

Sven Svebak describes the long way his Sense of Humor Questionnaire (SHQ) took, starting as an instrument for separating subjects in laughter experiments conducted as part of his master's thesis in 1969, via two published revisions in 1974, to its latest six-item revision in 1996. The latter revision (SHQ-6) turned the SHQ into a short scale which is economical when used in surveys. He reviews the early literature on humor assessment and outlines the ideas that led him to the revisions of the scale when they were first published more than 20 years ago. A final section includes a preliminary review of the empirical basis for the SHQ-6 revision, as well as its psychometrical support and ideas that helped to investigate the validity of this recent revision.

The study by Köhler and Ruch investigates different aspects of the validity of humor inventories. The psychometric property of validity relates to the extent to which an instrument actually measures what it claims to measure. The use of both questionnaire and behavioral assessments of the same or highly similar constructs (i.e., humor appreciation and humor creation) allows an evaluation of the extent to which these assessment approaches overlap. The results show that - cautiously phrased - the 
two approaches are not interchangeable. The second aim is to investigate the dimensions underlying the current sense of humor scales. Despite the high number of scales used, two dimensions seem to be sufficient to account for a high proportion of the reliable variance. This suggests that there is a high redundancy among the existing scales and that further attempts at measuring aspects of the sense of humor should concentrate on other, yet unexplored or underexplored domains of humor. Finally, the different measures of humor are related to personality and suggest different locations for the different dimensions of humor. On the whole, this presentation directs attention to technical aspects of scale construction and validation and also deals with content-related questions.

There was more work associated with preparing this special issue than I expected. For example, I initially considered including a broader variety of new and already published scales to more adequately represent what is out there in the literature. However, the reviewers apparently applied even higher quality criteria than I had been willing to when screening for potential contributions, and hence this issue ended up with a lower number of articles than originally intended. I have to admit that I was surprised by the fact that apparently many more share the awareness of need for quality with respect to measurement issues. Overall, this awareness makes me very optimistic for the future developments in our field.

Are there any recommendations for future approaches to the measurement of the "sense of humor"?

First, we do not necessarily need more new scales (although high quality instruments will be more than welcome), but we need more theoretical and empirical work on the definition or foundation of the concepts. However, deriving the construct, constructing the scale, and attempting to validate it in one single step (or even in one sample of subjects) do not seem to be all that fruitful. Each stage needs greater attention. Second, new (narrow) measurement devices should perhaps focus on construct areas not yet tapped into. For example, we do not yet have instruments which explicitly focus on aspects like humor as a benevolent world view, which tolerates and acceptingly smiles at the shortcomings of life and fellow-creatures. Likewise, do we have instruments that predict destructive forms of humor? Third, we should broaden our range of methodological approaches again; in the last years we seem to be focusing too much on 
self-report scales at the expense of behavioral observations, performance tests, peer-nomination, or peer-evaluations, biographical data, and others. Finally, the comprehensive definition of the sense of humor still remains the supreme but yet unattained goal. While the HBQD attempts to achieve this goal for the domain of reports of everyday humorous conduct, we do not have comparable approaches for other domains (like the different classes of humorous material or the humor-related lexical corpus of different languages).

This renaissance of research on the sense of humor provides a unique chance for a breakthrough in the definition of and measurements for this elusive concept. Let us take this chance.

University of Düsseldorf

\section{Notes}

* The preparation of this manuscript was facilitated by a Heisenberg-fellowship awarded from the Deutsche Forschungsgemeinschaft (Ru 480/1-1). Correspondence should be addressed to the author at Department of Psychology, Heinrich-Heine-University of Düsseldorf, Universitätsstraße 1, 40225 Düsseldorf, Germany.

1. Given the high density of already established personality traits, one might argue that there is no need for a separate "sense of humor" construct (see Ruch 1994). While other traits may indeed predict and perhaps even explain individual differences in humor, they cannot describe them and subsequently do not allow direct assessment, which is necessary, for example, for measuring changes in the sense of humor as an effect of a training program.

\section{References}

Chapman, Anthony and Hugh C. Foot (eds.)

1976 Humour and Laughter: Theory, Research and Applications. London: Wiley.

Freud, Sigmund

1905 Der Witz und seine Beziehung zum Unbewußten. Wien: Deuticke.

Goldstein, Jeffrey H. and Paul E. McGhee (eds.)

1972 The Psychology of Humor. New York: Academic Press.

LaFave, Lawrence, Jay Haddad, and William A. Maesen

1976 Superiority, enhanced self-esteem, and perceived incongruity humor theory.

In Anthony J. Chapman and Hugh C. Foot (eds.) Humour and Laughter:

Ruch, Willibald

Theory, Research and Applications. London: Wiley, 159-171.

1994 Temperament, Eysenck's PEN system, and humor-related traits. Humor 7, 209-244.

Schmidt-Hidding, Wolfgang (ed.)

1963 Europäische Schlüsselwörter. Band I: Humor und Witz. München: Huber. 